\title{
Cáncer Oromaxilofacial en Niños: Parte II Tumores Odontogénicos y de Glándulas Salivales Malignos
}

\author{
Maxillofacial Cancer in Children: Part II Malignant \\ Odontogenic and Salivary Gland Tumors
}

Javiera Rehbein*; Francisca Becerra*; Alejandra Fernández"; Sven Niklander"; Maureen Marshall ${ }^{* *}$ \& Alfredo Esguep ${ }^{* * *+}$

REHBEIN, J.; BECERRA, F.; FERNÁNDEZ, A.; NIKLANDER, S.; MARSHALL, M. \& ESGUEP, A. Cáncer oromaxilofacial en niños: parte II tumores odontogénicos y de glándulas salivales malignos. Int. J. Odontostomat., 10(2):277-282, 2016.

RESUMEN: A nivel mundial, la información acerca de tumores malignos del territorio maxilofacial que afectan a niños es limitada. La mayoría de los resportes consiste principalmente en datos de la población adulta. Las neoplasias malignas originadas del aparato odontogénicos y glándulas salivales son lesiones que con cierta frecuencia pueden afectar a la población infantil. Los tumores odontogénicos malignos son entidades sumamente raras que, correspondiendo a menos del $5 \%$ del total de tumores odontogénicos. Los sarcomas odontogénicos, si bien son poco frecuentes, corresponden a los tumores odontogénicos malignos más comunes en la infancia. Las neoplasias malignas de glándulas salivales corresponden al 35-60\% de los tumores de gándulas salivales en la infancia, siendo el más común de ellos el carcinoma mucoepidermoide. En general, el pronóstico de estas entidades es positivo sobre todo si es acompañado de un diagnóstico oportuno. A pesar de la baja frecuencia que presenta este grupo de patologías, no es menos cierto que es necesario saber con precisión cuales son los tejidos orales desde los cuales se pueden originar neoplasias malignas en los niños y tener una breve referencia diferencial entre ellos.

PALABRAS CLAVE: neoplasias, pediatría, odontogénico, cáncer.

\section{INTRODUCCIÓN}

Los tumores odontogénicos corresponden a un tipo infrecuente de lesiones que representan menos del $4 \%$ de todas las patologías orales y maxilofaciales. Los tumores odontogénicos pueden dividirse en benignos y malignos, estos últimos clasificándose según su origen en carcinomas y sarcomas odontogénicos. Los tumores odontogénicos malignos son extramandamente raros, abarcando aproximadamente el $5 \%$ del total de tumores odontogénicos. Los carcinomas odontogénicos suelen afectar más a personas adultas (sobre 50 años), mientras que los sarcomas odontogénicos a niños y adolescentes (Lai et al., 2012).

Los sarcomas odontogénicos son extremadamente raros y representan menos del $4 \%$ de todos los tumores odontogénicos (Wang et al., 2011). Co- rresponden a la contraparte maligna de los tumores odontogénicos que presentan ectomesénquima odontogénico (Barnes et al., 2005). Según la Organización Mundial de la Salud (OMS), están representados por dos entidades: Fibrosarcoma ameloblástico (FSA) y Fibrodentinosarcoma ameloblástico (FDSA) o Fibro-odontosarcoma ameloblástico (FOSA), dependiendo del tipo de tejido duro formado. De este modo la OMS distingue sarcomas odontogénicos desprovistos de tejido dental duro (FSA) de los que presentan evidencia focal de dentinoide o dentinoide más pseudoesmalte (FDSA/FOSA) (Wang et al.).

Las neoplasias de glándulas salivales pueden originarse a partir del epitelio o de los elementos mesenquimales que conforman los componentes no parenquimatosos de estas glándulas (Barnes et al.).

* Cirujano Dentista, Viña del Mar, Chile.

* Cirujano Dentista, Docente Cátedra de Patología, Universidad Andrés Bello, Viña del Mar, Chile.

${ }^{* * *}$ Profesor titular Departamento de Cirugía y Patología, Facultad de Odontología, Universidad Andrés Bello, Viña del Mar, Chile. 
Constituyen entre $3-5,5 \%$ de todas las neoplasias del organismo en la población pediátrica y de estos los malignos representan aproximadamente entre 35-60 $\%$ (da Cruz Perez et al., 2004). Presentan un comportamiento clínico similar al de los adultos, son más frecuentes en la parótida y en el sexo femenino. En cuanto a los tipos histológicos se pueden observar en la Tabla I (Barnes et al.). Los tipos más frecuentes son el carcinoma mucoepidermoide, seguido por el carcinoma de célula acinares y el carcinoma adenoide quístico. El tratamiento es principalmente quirúrgico y el pronóstico depende de la invasión a otros tejidos y del grado histológico del tumor (Ribeiro et al., 2002).

Aportando al conocimiento de las neoplasias malignas infantiles en el territorio oral y maxilofacial, a continuación se describe la epidemiología, etiopatogenia, tratamiento y pronóstico de los tipos más frecuentes de tumores malignos odontogénicos y de glándulas salivales que afectan a niños. Este apartado corresponde a la segunda parte sobre cáncer oromaxilofacial en niños.

I. Tumores Odontogénicos Malignos. En este apartado nos referiremos sólo a los tumores odontogénicos malignos de origen mesenquimal, ya que como se mencinó anteriormente los de origen epitelial no suelen afectar a niños. Algunos autores han considerado al FA, FDSA y al FOSA como una sólo variedad, sin embargo las OMS las considera lesiones independientes, por lo que se seguirá la clasificación propuesta por la OMS (Tabla I).

a) Fibrosarcoma ameloblástico. El FSA es un tumor odontogénico maligno raro, hasta la fecha sólo existen alrededor de 91 casos publicados. El tumor está formado por un componente epitelial ameloblástico benigno y otro ectomesenquimal maligno. Es considerado como la contraparte maligna del fibroma amelobástico (FA) (Lai et al.; Bregni et al., 2001). Su etiología es incierta. Se describe que puede presentarse sin lesiones preexistentes, o bien ser el resultado de una transformación maligna de un FA. Se estima que esto ocurre en un tercio de los FSA y está reportado que hasta un $11 \%$ de los FA sufrirán transformación maligna hacia un FSA (Jundt \& Reichart, 2008). Aproximadamente el $63 \%$ de los casos se registra en hombres y un $37 \%$ en mujeres (Zabolinejad et al., 2008). El rango de edad fluctúa entre los 3 y 89 años, con un promedio de 27,5 años (Barnes et al.).

Presenta un comportamiento biológico localmente agresivo con bajo potencial de metastizar. Sue- len manifestarse como aumentos de volumen dolorosos, a veces ulcerados, generalmente en la zona posterior de la mandíbula. No es raro que se asocien a parestesia (Jundt \& Reichart; Hu et al., 2014). Radiográficamente suelen manifestarse como una radoilucides uni o multilocular con márgenes poco definidos. El tratamiento de elección es la resección quirúrgica con márgenes de segurida. Como alternativa se puede usar quimio o radioterapia, pero no han mostrado ser eficazes. Algunos autores proponen que el FSA debiese ser considerado como un fibrosarcoma de bajo grado, en virtud de su comportamiento menos agresivo y pronóstico más favorable en comparación con otros fibrosarcomas (Akinyamoju et al., 2013; Barnes et al.). Presenta una baja tendencia a recurrir $(23,9 \%)$ y la tasa de mortalidad es baja $(25,4 \%)$ (Lai et al.).

b) Fibrodentinosarcoma ameloblástico y Fibroodontosarcoma ameloblástico. EI FDSA y FOSA, son neoplasias con características histológicas de fibrosarcoma ameloblástico, junto con displasia dentinaria y/o esmalte/pseudoesmalte y dentina/ dentinoide respectivamente. Se propone que el FOSA surgiría a partir de de un Fibroodontoma ameloblástico. Se pueden presentar entre los 12 y 83 años, estimándose un peak en la tercera década, una década posterior al promedio de edad de la presentación del precursor benigno. En lo que respecta a su distribución topográfica, la mandíbula es la más afectada, comúnmente en el sector posterior (Lai et al.; Barnes et al.).

El comportamiento clínico e histopatológico, tratamiento de elección y tasa de mortalidad de estas entidades es igual que el FSA.

II. Tumores Malignos de las Glándulas Salivales. A continuación se presentan exclusivamente las neoplasias malignas de glándulas salivales más frecuentes en el territorio oral y maxilofacial en la población pediátrica. Estas formas son: carcinoma mucoepidermoide, carcinoma de células acínicas y carcinoma adenoide quístico (Ribeiro et al.).

a) Carcinoma mucoepidermoide. El carcinoma mucoepidermoide (CME) es un tumor epitelial glandular maligno que está formado por células mucosas, epidermoide, intermedia, columnares y claras. Es el tumor de glándulas salivales más frecuente en niños (Védrine et al., 2005). Según Rahbar et al., aproximadamente un $50 \%$ de los tumores de glándulas salivales en el grupo pediátrico corresponden a este tumor (Rahbar et al., 2006). Védrine et al., plantean 
Tabla I. Clasificación de los tumores odontogénicos malignos (Barnes et al., 2005).

\begin{tabular}{ll}
\hline \multicolumn{1}{c}{ Carcinomas Odontogénicos } & \multicolumn{1}{c}{ Sarcomas Odontogénicos } \\
\hline Ameloblastoma (Maligno) Metastatizante & Fibro Sarcoma Ameloblástico \\
Carcinoma Ameloblástico tipo primario & Fibrodentino- Fibro Odonto Sarcoma \\
$\begin{array}{l}\text { Carcinoma Ameloblástico tipo secundario } \\
\text { (desdiferenciado) Intraóseo }\end{array}$ & Ameloblástico \\
Carcinoma Ameloblástico tipo secundario & \\
(desdiferenciado) Periférico & \\
Carcinoma de Células Escamosas Intraóseo & \\
Primario-tipo Sólido & \\
Carcinoma de Células Escamosas Intraóseo & \\
Primario derivado de tumor odontogénico & \\
queratoquístico & \\
Carcinoma de células escamosas Intraóseo & \\
primario derivado de quiste odontogénico & \\
Carcinoma Odontogénico de células claras & \\
Carcinoma Odontogénico de células fantasma & \\
\hline
\end{tabular}

que los CME de glándulas salivales representan un $0,08 \%$ de las neoplasias malignas en los niños y que adicionalmente, sólo el $5 \%$ de los CME de glándulas salivales se presentan durante la infancia, y ocasionalmente antes de los 10 años. El rango de edad estimado a presentarse este tumor es entre los 5 meses y 5 años de edad (Rahbar et al.). Presenta predilección por el sexo femenino y afecta principalmente a la glándula parótida (70 \%) (Perez et al., 2008), donde el lóbulo superficial se ve comprometido en un $75 \%$ de los casos (Védrine et al.), y en menor medida la glándula submandibular y las glándulas salivales menores. Cuando afecta a estas últimas, la ubicación más común es el paladar, aunque también pueden verse afectadas otras zonas como los labios, la mucosa oral y el área retromolar. Perez et al., señalan que cuando los CME afectan a las glándulas salivales menores en este grupo etario tienen una frecuencia similar o incluso superior a la del adenoma pleomorfo.

Jen et al. (2002) realizaron una revisión de la literatura entre los años 1957-2000 y resumieron todos los casos reportados de CME en niños. Aunque la mayoría de la información sobre el sexo y la edad de los pacientes no estaban disponibles en la mayoría de los artículos revisados, trataron de analizar los datos según la información a su alcance. El número total de pacientes pediátricos (menores de 20 años de edad) con CME fue de 212. Entre ellos, 173 (82 \%) estaban ubicados en la glándula parótida, 8 (4 \%) en la glándula submandibular y 31 (14 \%) en las glándulas salivales menores. Entre las glándulas salivales menores, el sitio de ubicación más frecuente fue el paladar. La edad promedio de los pacientes fue de 13 años de edad. La mayoría de los casos en que la parótida se encontraba afectada fueron detectados entre los 11 y 15 años de edad, mientras que sólo 2 casos fueron vistos en niños menores de 4 años.

En un estudio por Ceceñas et al. (2010) sobre el periodo comprendido entre los años 1998 y 2009 , de un total de 122.291 estudios quirúrgicos, sólo 11 casos se presentaron en pacientes pediátricos. De este grupo un $45,5 \%$ fueron benignos y un $54,5 \%$ malignos. El tumor maligno más frecuente fue el CME (27\%). La glándula parótida fue la localización más frecuente (73\%), seguida de la glándula submaxilar (27\%). No se encontraron casos que afectaran a la glándula sublingual ni a las glándulas salivales menores. En cuanto a la edad, el $81,8 \%$ de los casos se presentaron en pacientes mayores de 10 años de edad, y el $36,3 \%$ en pacientes entre 15 y 18 años de edad.

Su etiopatogenia es desconocida (Perez et al.). Se postula que proviene de una reserva de células pluripotenciales de los conductos excretores de las glándulas salivales, las cuales poseen la capacidad de diferenciarse en células escamosas, columnares y mucosas. Adicionalmente, se sugiere que la exposición a radiación ionizante podría ser un factor etiológico asociado (Rahbar et al.). Védrine et al., reportaron que 11 de 18 casos de pacientes juveniles afectados por CME presentaban historia previa de tratamiento con radioterapia o quimioterapia a consecuencia de un tumor primario, específicamente leucemia y linfoma. 
b) Carcinoma de células acínicas. Es una neoplasia maligna epitelial de las glándulas salivales en la que al menos una de las células neoplásicas presenta diferenciación serosa de células acinares, las que se caracterizan por presentar citoplasmas que presentan gránulos secretores de zimógenos. Las células ductales también componen esta neoplasia. Se cree que esta neoplasia proviene de células progenitores de los ductos intercalares del tejido salival (Barnes et al.).

En relación a su epidemiología es poco frecuente, pero es el segundo tumor maligno más común de las glándulas salivales en los niños menores de 12 años de edad, reportándose más frecuentemente en mujeres (Leuin et al., 2007). Tanto Jones et al. (1997), y Leuin et al., reportaron casos de niños menores de 13 años de edad diagnosticados con carcinoma de células acínicas, todos ubicados en la parótida. Su prevalencia se ha reportado entre un $13 \%$ y $34 \%$ del total de tumores de glándulas salivales malignos en niños (Jones et al.).

Cuando afecta la parótida el tratamiento consiste en parotidectomía (Sato et al., 2005). Aparentemente, los resultados del tratamiento para esta neoplasia en la glándula parótida son buenos. Los rangos de sobrevida van de un $85 \%$ a un $96 \%$. Sin embargo, a veinte años decrecen en un $56 \%$. En aquellos pacientes en los que no se lleva a cabo un tratamiento adecuado la recurrencia de esta enfermedad puede alcanzar un porcentaje de 25-50 \% (Leuin et al.).

c) Carcinoma adenoide quístico. Es un tumor epitelial basaloide que consiste en células epiteliales y mioepiteliales en configuraciones morfológicas variables, incluyendo los patrones tubular, cribiforme y sólido. Presenta un curso clínico implacable y por lo general desencadena un desenlace fatal. Representa aproximadamente un $10 \%$ de todas las neoplasias de glándulas salivales y un $30 \%$ de los tumores de glándulas salivales menores. Aparentemente no presenta predilección por sexo. Puede presentarse en todos los grupos etarios, con una mayor incidencia en la edad media y avanzada. Involucra frecuentemente a la glándula parótida, submandibular y glándulas salivales menores, de preferencia en el paladar, seguido de la lengua, mucosa oral, labios y piso de boca (Barnes et al.).

A nivel mundial, la información a cerca de tumores malignos en niños que afectan al territorio maxilofacial es limitada, consistiendo principalmente en datos de la población adulta. Consultamos las bases de datos de PubMed, CEN-TRAL y EBSCO y la mayoría de los estudios encontrados se refieren a casos de tumores orales y maxilofaciales específicos, y otros suelen agrupar casos pediátricos junto con los casos de adultos. A pesar de la baja frecuencia que presenta este grupo de patologías, no es menos cierto que es necesario saber con precisión cuales son los tejidos orales desde los cuales se pueden originar neoplasias malignas en los niños y tener una breve referencia diferencial entre ellos.

Es difícil abordar el tema desde el punto de vista de una revisión bibliográfica, ya que no se cuenta con estudios sistematizados de todas las manifestaciones posibles de cáncer en la cavidad oral al margen de su poca frecuencia. Esto dificulta el análisis epidemiológico, que actualmente permite entender los patrones de desarrollo de cada una de las neoplasias y establecer políticas orientadas al fomento de la prevención y diagnóstico precoz.

Datos tales como incidencia, prevalencia, tipos y distribución de estas lesiones según edad, género, localización anatómica, entre otras variables, provienen de estudios aislados, por lo que es incierto si las series disponibles de estas patologías reflejan de manera fidedigna tanto su verdadera naturaleza como sus características clinicopatológicas, por lo que no es posible hacer consideraciones definitivas en torno a ello.

Además, al estudiar este grupo de lesiones es importante considerar la distribución geográfica. La mayoría de países occidentales reportan una baja incidencia de tumores malignos orales en el grupo pediátrico. Por otro lado, en África, se observa una mayor prevalencia de tumores malignos, lo que se atribuye a un elevado porcentaje de linfomas, asociado probablemente a la alta prevalencia de malaria, VIH y virus Epstein Barr. Esta variación también puede ser aplicada a las distintas zonas de nuestro país, tema relevante de ser estudiado. Cabe destacar que en Chile no existen estadísticas sobre la casuística de estas patologías a nivel oral y maxilofacial en los niños. En la última publicación del MINSAL existen alusiones sobre cáncer oral en el grupo pediátrico, donde mencionan principalmente el rabdomiosarcoma, linfoma de Hodgkin y la histiocitosis. Desde un punto de vista especializado es necesario tener una clara idea de todas las pontencialidades cancerológicas de la boca. Por otro lado, para favorecer su diagnóstico creemos necesario que los laboratorios de histopatología cuenten con herramientas inmunohistoquímicas de marcadores tumorales y también abrir la posibilidad de establecer relaciones con laboratorios de citogenética e imagenología. 
El amplio espectro de patologías encontradas requiere de atención y estrecha colaboración entre pediatras oncólogos, radioterapeutas, patólogos orales y odontólogo, todos ellos especializados en el área de cabeza y cuello.
Esperamos que esta revisión constituya un instrumento que permita mejorar el conocimiento de estas lesiones y que sea un complemento a la guía: Cuando sospechar de un cáncer en el niño y cómo derivar, publicada por el MINSAL el año 2011.

REHBEIN, J.; BECERRA, F.; FERNÁNDEZ, A.; NIKLANDER, S.; MARSHALL, M. \& ESGUEP, A. Maxillofacial cancer in children: Part II malignant odontogenic and salivary gland tumors. Int. J. Odontostomat., 10(2):277-282, 2016.

ABSTRACT: Globally, information about the maxillofacial malignant tumors affecting children is limited. Most reported data consists mainly of studies in the adult population. Malignant neoplasms arising from odontogenic apparatus and salivary glands are lesions that frequently can affect children. Malignant odontogenic tumors are extremely rare entities, corresponding to less than $5 \%$ of all odontogenic tumors. Odontogenic sarcomas, although they are rare, correspond to the most common malignant odontogenic tumors in childhood. Malignant salivary gland neoplasms correspond to $35-60 \%$ of tumors of salivary glands during childhood and the most common of these is mucoepidermoid carcinoma. In general, the prognosis of these entities is positive especially when there is a timely diagnosis. Despite the low frequency presented by this group of diseases, the fact remains that it is necessary to know precisely what the originating oral tissues are which can cause malignancies in children and have a brief reference differential between them.

KEY WORDS: neoplasias, pediatrics, odontogenic cancer.

\section{REFERENCIAS BIBLIOGRÁFICAS}

Akinyamoju, A. O.; Olusanya, A. A.; Adeyemi, B. F. \& Kolude, B. Ameloblastic fibrosarcoma: Report of a case. J. Oral Maxillofac. Pathol., 17(3):424-6, 2013.

Barnes, L.; Eveson, J. W.; Reichart, P. \& Sidransky, D. Pathology and Genetics of Head and Neck Tumours. In: Kleihues, P. \& Sobin, L. H. (Eds.). World Health Organization Classification of Tumours. World Health Organization, International Agency for Research on Cancer (IARC). Lyon, IARC Publications, 2005.

Bregni, R. C.; Taylor, A. M. \& García, A. M. Ameloblastic fibrosarcoma of the mandible: report of two cases and review of the literature. J. Oral Pathol. Med., 30(5):31620, 2001.

Ceceñas, F. L. A.; Rivera, L. O. S.; Galdamez, T. A.; Áncer, R. J. \& Barboza, Q. O. Tumores epiteliales de glándulas salivales en la población pediátrica del Hospital Universitario Dr. José E González. Estudio retrospectivo de 11 años. Patol. Rev. Latinoam., 48(4):234-7, 2010.

da Cruz Perez, D. E.; Pires, F. R.; Alves, F. A.; Almeida, O. P. \& Kowalski, L. P. Salivary gland tumors in children and adolescents: a clinicopathologic and immunohistochemical study of fifty-three cases. Int. J. Pediatr. Otorhinolaryngol., 68(7):895-902, 2004.

Hu, Y. Y.; Deng, M. H.; Yuan, L. L. \& Niu, Y. M. Ameloblastic fibrosarcoma of the mandible: A case report and mini review. Exp. Ther. Med., 8(5):1463-6, 2014.
Jen, K. Y.; Cheng, J.; Maruyama, S.; Hayashi, T.; Suzuki, I.; Shingaki, S. \& Saku, T. Mucoepidermoid carcinoma in children: Report of a case and review of literature. Oral. Med. Pathol., 7(1):27-31, 2002.

Jones, A. O.; Lam, A. H. \& Martin, H. C. Acinic cell carcinoma of the parotid in children. Australas. Radiol., 41(1):448, 1997.

Jundt, G. \& Reichart, P. A. Maligne odontogene tumoren. Pathol., 29(3):205-13, 2008.

Lai, J.; Blanas, N.; Higgins, K. \& Klieb, H. Ameloblastic fibrosarcoma: report of a case, study of immunophenotype, and comprehensive review of the literature. J. Oral Maxillofac. Surg., 70(8):2007-12, 2012.

Leuin, S. C.; Cunningham, M. J.; Curtin, H. \& Faquin, W. C. Childhood acinic cell carcinoma of the accessory parotid gland: A rare combination. Int. J. Pediatr. Otorhinolaryngol., 2(1):1-5, 2007.

Perez, D. E.; Pires, F. R.,.; Alves, F. A.; Lopes, M. A.; de Almeida, O. P. \& Kowalski, L. P. Juvenile intraoral mucoepidermoid carcinoma. J. Oral Maxillofac. Surg., 66(2):308-11, 2008.

Rahbar, R.; Grimmer, J. F.; Vargas, S. O.; Robson, C. D.; Mack, J. W.; Perez-Atayde, A. R.; Marcus, K. J.; Grier, H. E.; Healy, G. B. \& McGill, T. J. Mucoepidermoid carcinoma of the parotid gland in children: A 10-year 
experience. Arch. Otolaryngol. Head Neck Surg., 132(4):375-80, 2006.

Ribeiro, K. C.; Kowalski, L. P.; Saba, L. M. \& de Camargo, B. Epithelial salivary glands neoplasms in children and adolescents: a forty-four-year experience. Med. Pediatr. Oncol., 39(6):594-600, 2002.

Sato, T.; Kamata, S. E.; Kawabata, K.; Nigauri, T.; Mitani, H.; Beppu, T. \& Sato, M. Acinic cell carcinoma of the parotid gland in a child. Pediatr. Surg. Int., 21(5):377-80, 2005.

Védrine, P. O.; Coffinet, L.; Temam, S.; Montagne, K.; Lapeyre, M.; Oberlin, O.; Orbach, D.; Simon, C. \& Sommelet, D. Mucoepidermoid carcinoma of salivary glands in the pediatric age group: 18 clinical cases, including 11 second malignant neoplasms. Head Neck, 28(9):827-33, 2006.

Wang, S.; Shi, H.; Wang, P. \& Yu, Q. Ameloblastic fibroodontosarcoma of the mandible: imaging findings. Dentomaxillofac. Radiol., 40(5):324-7, 2011.

Zabolinejad, N.; Hiradfar, M.; Anvari, K. \& Razavi, A. S. Ameloblastic fibrosarcoma of the maxillary sinus in an infant: a case report with long-term follow-up. J. Pediatr. Surg., 43(2):e5-8, 2008.
Dirección para Correspondencia:

Alfredo Esguep Sarah

Avenida Valparaíso 1560

Viña del Mar

CHILE

Email: aesguep@unab.cl

Recibido : 22-07-2015

Aceptado: 11-04-2016 